\title{
Alkenes as Azido Precursors for the One-Pot Synthesis of 1,2,3- 2Triazoles Catalyzed by Copper Nanoparticles on Activated Carbon
}

\author{
${ }_{3}$ Francisco Alonso, ${ }^{\dagger} *$ Yanina Moglie, ${ }^{\dagger}$ Gabriel Radivoy, ${ }^{\dagger}$ and Miguel Yus ${ }^{\dagger}$ \\ $4{ }^{\dagger}$ Departamento de Química Orgánica, Facultad de Ciencias and Instituto de Síntesis Orgánica (ISO), Universidad de Alicante, Apdo \\ 5 99, 03080 Alicante, Spain \\ $6{ }^{\star}$ Departamento de Química, Instituto de Química del Sur (INQUISUR-CONICET), Universidad Nacional del Sur, Avenida Alem \\ 7 1253, 8000 Bahía Blanca, Argentina
}

8 S Supporting Information

9 ABSTRACT: A one-pot protocol for the synthesis of 1,2,3-

10 triazoles has been developed starting from inactivated alkenes

11 and based on two click reactions: the azidosulfenylation of the

12 carbon-carbon double bond and the copper-catalyzed azide-

13 alkyne cycloaddition (CuAAC). High yields of the $\beta$ -

14 methylsulfanyl triazoles have been attained using $\mathrm{CuNPs} / \mathrm{C}$

15 as catalyst, with other commercial copper catalysts being completely inactive. The versatility of the methylsulfanyl group has been

16 demonstrated through a series of synthetic transformations, including direct access to 1-vinyl and 4-monosubstituted triazoles.

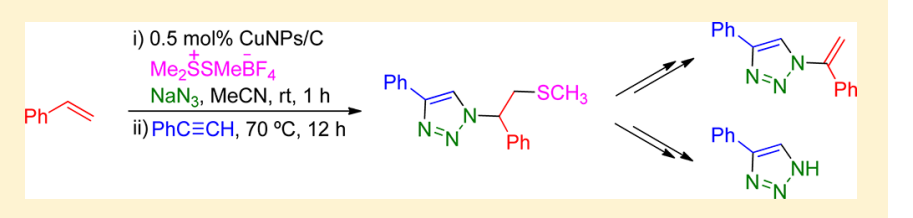

17 lick chemistry has become one of the most important 18 concepts in modern chemistry. ${ }^{1}$ It represents certain 19 highly efficient and reliable reactions which are modular, wide 20 in scope, high yielding, stereospecific, and proceed under 21 simple and benign conditions with straightforward procedures 22 for product isolation. Recently, click chemistry's first decade has 23 been celebrated, ${ }^{2}$ with an endless list of disciplines having 24 benefited from the unique advantages offered by this type of 25 reaction. The copper-catalyzed azide-alkyne cycloaddition $26(\mathrm{CuAAC})^{3}$ fulfills the aforementioned series of rigorous criteria, 27 as defined by Sharpless et al., turning this reaction into the click 28 reaction by antonomasia. ${ }^{4}$ The nucleophilic opening of spring29 loaded rings (i.e., epoxides, aziridines, cyclic sulfates, cyclic 30 sulfamidates, aziridinium ions, and episulfonium ions) also 31 belongs to the privileged list of click reactions because they are 32 reliable, stereospecific, often highly regioselective, and nearly 33 quantitative. ${ }^{1}$

34 The CuAAC has been traditionally implemented with 35 preformed organic azides. More advantageous are, however, 36 the methodologies in which the organic azides are generated in 37 situ from organic halides ${ }^{5}$ (three-component azide-alkyne 38 cycloaddition) because (a) hazards derived from their isolation 39 and handling are minimized, (b) time-consuming and waste40 generating additional synthetic steps are avoided, and (c) the 41 common organic solvents utilized (e.g., dioxane, toluene, DMF, 42 dichloromethane, and hexane) can be replaced by neat water. 43 In this vein, efforts have been recently devoted to develop new 44 catalytic systems which allow the CuAAC from other azide 45 precursors, namely amines, ${ }^{6}$ tosylates, ${ }^{7}$ diarylidodonium salts, ${ }^{8}$ 46 epoxides, ${ }^{9}$ alcohols, ${ }^{10}$ and boronic acids. ${ }^{11}$ Favi et al. reported 47 the one-pot copper(II)-catalyzed aza-Michael addition of 48 trimethylsilyl azide to 1,2-diaza 1,3-dienes and copper(I)49 catalyzed 1,3-dipolar cycloaddition of the in situ generated $\alpha$ 50 azido hydrazones with alkynes. ${ }^{12}$ However, alkenes are the most commonly available starting materials which can provide a 51 carbon framework. To the best of our knowledge, the synthesis 52 of 1,2,3-triazoles from inactivated alkenes has never been 53 described.

On the other hand, there is an upsurge of interest in the use 55 of nanostructured copper catalysts for CuAAC because of their 56 large surface-to-volume ratio, varied morphology, and sustain- 57 able catalytic applications. ${ }^{13}$ Owing to our dedication to study 58 and understand the reactivity of metal colloids, ${ }^{14}$ we found out 59 that active copper [obtained from $\mathrm{CuCl}_{2} \cdot 2 \mathrm{H}_{2} \mathrm{O}$, lithium metal, 60 and a catalytic amount of 4,4'-di-tert-butylbiphenyl (DTBB) in 61 THF at room temperature] was able to reduce different organic 62 functionalities under very mild conditions. ${ }^{15}$ We also 63 discovered that copper nanoparticles (CuNPs) are formed 64 when the active copper is generated from anhydrous $\mathrm{CuCl}_{2} 65$ under the above-mentioned conditions. These unsupported 66 copper nanoparticles (10 mol \%) effectively catalyzed the 67 CuAAC in the presence of triethylamine at $65{ }^{\circ} \mathrm{C}$ in THF. ${ }^{16} 68$ Remarkably short reaction times (10-120 min), comparable to 69 those previously reported under microwave heating, were 70 recorded in the absence of any stabilizing additive or ligand. 71 Unfortunately, the CuNPs underwent dissolution under the 72 reaction conditions which precluded their reuse. More recently, 73 we introduced a catalyst consisting of oxidized copper 74 nanoparticles on activated carbon (CuNPs/C), readily 75 prepared under mild conditions, which exhibited a high 76 versatility in the multicomponent click synthesis of 1,2,3- 77 triazoles in water. ${ }^{17}$ Not only organic halides but diazonium 78 salts, anilines, and epoxides were successfully used as azide 79 precursors in the CuAAC (Scheme 1). We want to present $80 \mathrm{~s} 1$ herein the first one-pot transformation of inactivated olefins 81

Received: January 17, 2013 
Scheme 1. Multicomponent Synthesis of 1,2,3-Triazoles from Different Azide Precursors Catalyzed by CuNPs/C in Water

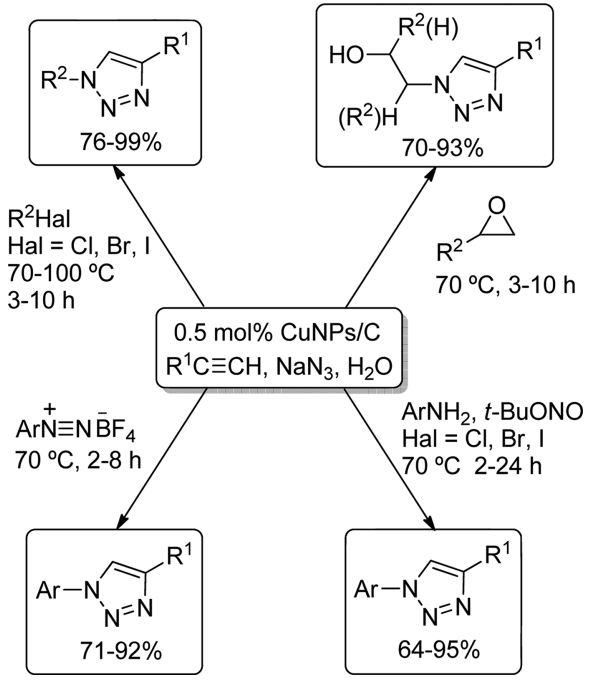

82 into 1,2,3-triazoles by taking advantage of two consecutive click 83 reactions: (a) the ring-opening of in situ generated 84 episulfonium ions by the azide anion and (b) the reaction of 85 the in situ generated azides with alkynes catalyzed by CuNPs/ $86 \mathrm{C}$.

87 We envisaged the potential transformation of alkenes into 88 triazoles inspired by the azasulfenylation of alkenes developed 89 by Trost et al. ${ }^{18}$ In this methodology, an alkene was treated 90 with dimethyl(methylthio)sulfonium tetrafluoroborate 91 (DMTSF $)^{19}$ at $0{ }^{\circ} \mathrm{C}$ to room temperature, followed by the 92 addition of a nitrogen nucleophile at room temperature and 93 stirring for 1-4 days. After an optimization of the reaction 94 conditions (i.e, solvent, catalyst, temperature, and reaction 95 time) we discovered a more convenient variation of this 96 method in which the alkene was directly mixed with CuNPs/C, 97 DMTSF, and $\mathrm{NaN}_{3}$ in $\mathrm{MeCN}$ to produce the corresponding 98 methylsulfanyl azide in only $1 \mathrm{~h}$ at room temperature; 99 apparently, the CuNPs accelerate this process. The subsequent 100 reaction with the alkyne represents, to the best of our 101 knowledge, is the first example of triazole synthesis from an 102 inactivated alkene in one pot (Scheme 2).

Scheme 2. Optimized Conditions for the One-Pot Synthesis of Triazoles from Alkenes Catalyzed by CuNPs/C

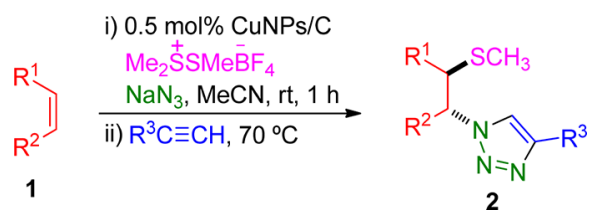

103 With this methodology in hand, a series of representative 104 alkenes and different alkynes were subjected to this one-pot 105 consecutive double-click protocol (Table 1). We first studied 106 the reaction of cyclohexene with various electronically different 107 alkynes (Table 1, entries 1-5). Good yields were recorded for 108 the electronically neutral alkynes phenylacetylene (2a) and $p$ 109 tolylacetylene $(\mathbf{2 b})$ as well as for the electronically rich and 110 poor 4-methoxyphenylacetylene (2c) and 4-(trifluoromethyl)111 phenylacetylene (2d), respectively (Table 1, entries $1-4)$. The aliphatic alkyne oct-1-yne was found to be more reluctant to 112 react and needed prolonged heating in order to reach a yield 113 similar to those of the aromatic alkynes (Table 1, entry 5). 114 Interestingly, a high control was achieved in the mono- 115 azidosulfenylation of cycloocta-1,5-diene (1b). The subsequent 116 reaction with phenylacetylene $(\mathbf{2 a})$ gave rise to the product $3 \mathbf{b a} 117$ in excellent yield, which possesses a carbon-carbon double 118 bond available for further functionalization (Table 1, entry 6). 119 Very similar yields and reaction times as those in entry 6 were 120 noted when starting from the oxacyclic olefin 2,5-dihydrofuran 121 (1c) (Table 1, entry 7). It is noteworthy that the cyclic olefins 122 1a-c provided exclusively the trans-methylsulfanyl triazol-1-yl 123 products. These results are in agreement with the reaction 124 taking place through an episulfonium ion intermediate, which 125 undergoes trans-diaxial ring-opening through an $\mathrm{S}_{\mathrm{N}} 2$ process. 126 This is the same trend we observed in the synthesis of 1,2,3- 127 triazoles from cycloalkene oxides. ${ }^{17 c}$

128

We next studied the behavior of acyclic olefins in the title 129 reaction. The symmetrical internal alkene $(Z)$-oct-4-ene (1d), 130 when combined with phenylacetylene (2a), furnished 3da with 131 a $4 R^{*}, 5 R^{*}$ relative configuration proposed in view of the 132 aforementioned trend (Table 1 , entry 8 ). The azidosulfenyla- 133 tion of the terminal olefin oct-1-ene (1e) was found to be less 134 regioselective when compared with the azidolysis of oct-1-ene 135 oxide. ${ }^{17 \mathrm{c}}$ In this case, the CuAAC with phenylacetylene (2a) 136 yielded a ca. 3:1 mixture of regioisomers, the major one derived 137 from the attack of the azide ion to the less hindered position of 138 the intermediate episulfonium ion (Table 1, entry 9). The 139 stabilization of the partially developed positive charge on the 140 internal carbon atom of the episulfonium ion in the transition 141 state could account for the formation of the minor regioisomer 142 3ea'. Fortunately, the two regioisomers could be easily 143 separated by column chromatography. Triazoles $3 \mathrm{fa}$ and $3 \mathrm{fa}^{\prime}, 144$ derived from the unsymmetric cyclic olefin 1-methylcyclohex-1- 145 ene, were produced in a nearly 1:1 regioisomeric ratio and 146 could be also separated (Table 1, entry 10). Finally, when 147 styrene was subjected to the standard procedure, either with 148 phenylacetylene (2a) or oct-1-yne (2e), the expected triazoles 149 3 ga and 3ge where obtained, respectively, in good yields as 150 single regioisomers (Table 1, entries 11 and 12). In both cases, 151 attack of the azide ion to the internal carbon atom of the 152 intermediate episulfonium ion was preferred as it was also 153 previously observed in the domino azidolysis-CuAAC of 154 styrene oxide and phenylacetylene. ${ }^{17 \mathrm{c}}$ These results can be 155 explained by the partially developed positive charge during the 156 nucleophilic azide attack in the unsymmetrical ring-opening 157 transition state, which is more stabilized at the benzylic position 158 of the episulfonium ion. Recently, 1,2,3-triazoles have been 159 successfully applied in organic synthesis as ligands, ${ }^{20}$ with 160 compounds in Table 1 representing a new family of potential 161 N,S-triazolyl ligands.

162

Contrary to the good recycling behavior observed for 163 CuNPs/C in other multicomponent click reactions, ${ }^{17}$ in the 164 present case reutilization was inefficient, very probably due to 165 catalyst poisoning by sulfur. Nevertheless, this fact is not so 166 important if we take into account the low copper loading 167 deployed in the experiments $(0.5 \mathrm{~mol} \%)$. On the other hand, it 168 is our premise that any laboratory-made catalyst should be 169 more efficient than commercially available catalysts used for the 170 same purpose; otherwise, it is difficult to economically justify 171 the time, materials, and human resources employed during its 172 preparation. With this principle in mind, we undertook a 173 comparative study on the reactivity of CuNPs/C with some 174 
Table 1. One-Pot Click Synthesis of 1,2,3-Triazoles from Alkenes Catalyzed by CuNPs/C ${ }^{a}$

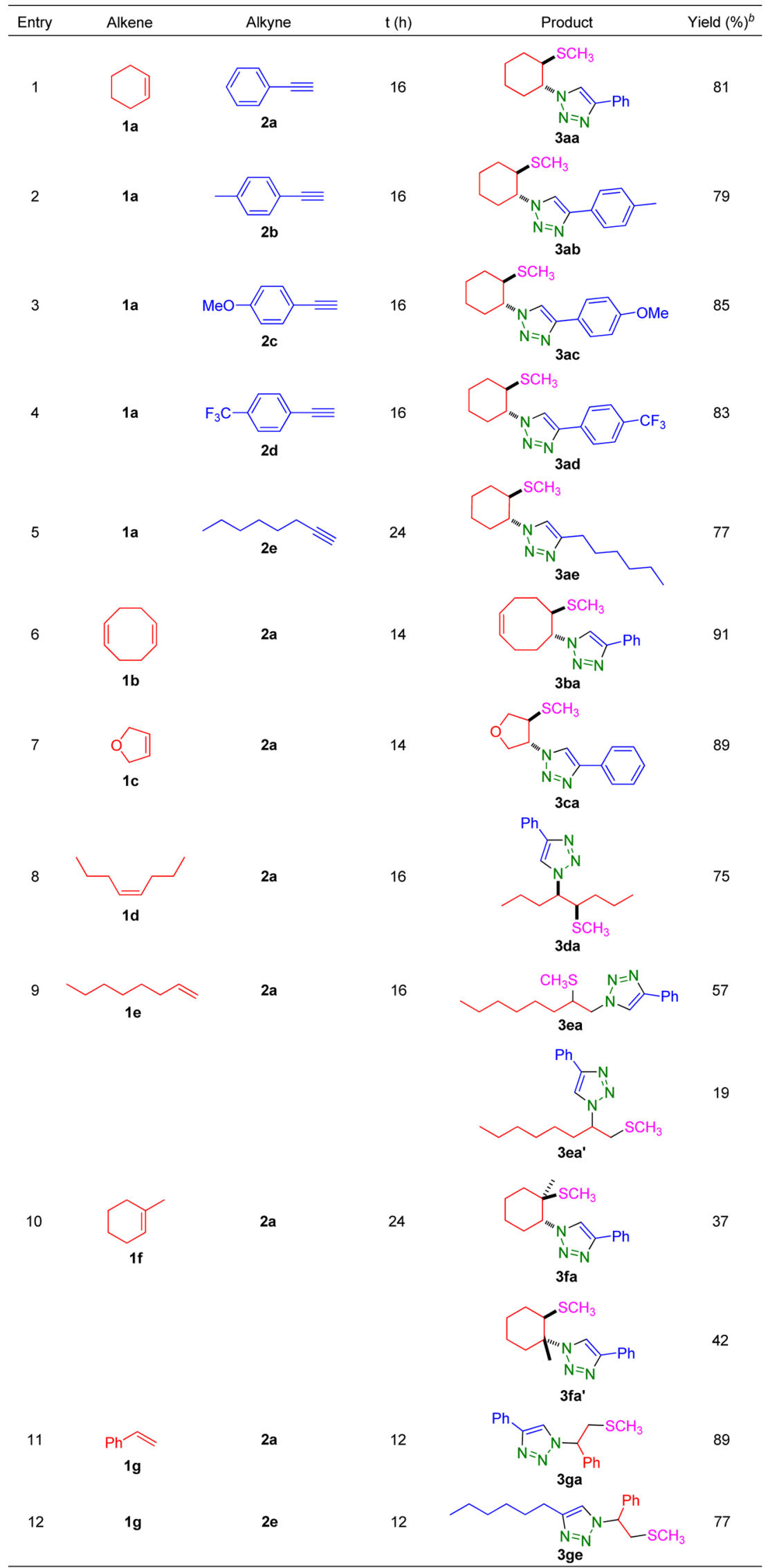

${ }^{a}$ Reagents and conditions: 1 (0.5 mmol), NaN $3(0.6 \mathrm{mmol}), \mathrm{DMTSF}(0.6 \mathrm{mmol}), \mathrm{CuNPs} / \mathrm{C}(0.5 \mathrm{~mol} \%), \mathrm{MeCN}(2 \mathrm{~mL}), \mathrm{rt}, 1 \mathrm{~h} ; 2$ (0.5 mmol), 70 ${ }^{\circ} \mathrm{C}$, time (h). ${ }^{b}$ Isolated yield. 
175 commercially available copper sources. The standard conditions 176 were applied to the reaction of cyclohexene (1a) with DMTSF, $177 \mathrm{NaN}_{3}$, and phenylacetylene (2a) leading to 3aa (Table 2). We

\section{Table 2. One-Pot Click Synthesis of 1,2,3-Triazoles from Alkenes Catalyzed by Different Copper Catalysts ${ }^{a}$}<smiles>C1=CCCCC1</smiles>

1a

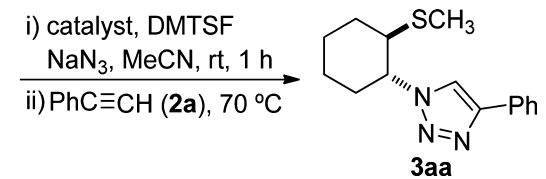

${ }^{a} 1 \mathrm{a}(0.5 \mathrm{mmol}), \mathrm{NaN}_{3}(0.6 \mathrm{mmol}), \operatorname{DMTSF}(0.6 \mathrm{mmol}), \mathrm{MeCN}(2$ $\mathrm{mL}) .{ }^{b}$ Isolated yield. ${ }^{c}$ Not detected.

178 were delighted to check that none of the commercial catalysts 179 was active in this transformation, where even the initial 180 azidosulfenylation step failed (Table 2, entries 1-5). In 181 contrast, the copper-nanoparticle supported catalyst produced 182 the desired product in good isolated yield (Table 2, entry 6). 183 These results are in agreement with the fact that CuNPs/C 184 could also catalyze the first synthetic step. This catalytic role 185 was clearly demonstrated by carrying out two experiments: (a) 186 the reaction of cyclohexene with DMTSF and $\mathrm{NaN}_{3}$ in $\mathrm{MeCN}$ 187 at $\mathrm{rt}(1-24 \mathrm{~h})$ gave a complex mixture of products, with the 188 expected azide representing only 5-24\%; (b) the same reaction 189 in the presence of $0.5 \mathrm{~mol} \% \mathrm{CuNPs} / \mathrm{C}$ provided that azide 190 quantitatively in only $1 \mathrm{~h}$ (see the Supporting Information).

191 Finally, we sought to capitalize on the presence of the 192 methylsulfanyl group to structurally modify the triazoles 3 193 (Scheme 3). A variety of conditions were tested in order to 194 achieve maximum selectivity, with the best results being shown

Scheme 3. Synthetic Transformations of Triazole 3ga

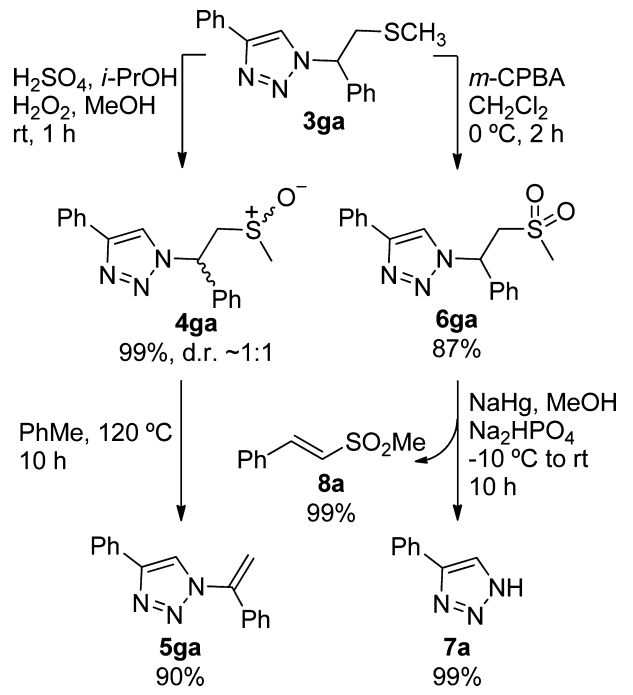

in Scheme 3. Oxidation of the parent triazole 3ga with 195 hydrogen peroxide was mild and fast giving a ca. 1:1 196 diastereomeric mixture of sulfoxide 4ga. Sulfoxide elimination 197 under thermal conditions led to the vinyltriazole 5ga in an 198 overall quantitative conversion. We must point out that the 199 synthesis of 1-vinyl-1,2,3-triazoles has been scarcely studied, ${ }^{21} 200$ with this method representing an effectual approach. Oxidation 201 of the parent triazole 3ga to the corresponding sulfone 6ga was 202 easily accomplished with $m$-CPBA. Treatment of 6 ga with 203 sodium amalgam in methanol afforded the 4-monosubstituted 204 triazole 7a together with methyl (E)- $\beta$-styryl sulfone (8a). 205 These experiments prove the versatility of the $\beta-206$ (methylsulfanyl)ethyl-substituted 1,2,3-triazoles 3 . 207

In conclusion, we have described the first one-pot synthesis 208 of 1,2,3-triazoles from inactivated alkenes through a sequence 209 including two click steps catalyzed by CuNPs/C: the 210 azidosulfenylation of the olefin and the reaction of the in situ 211 generated organic azide with the terminal alkyne. The $\beta-212$ methylsulfanyl triazoles, potential interesting ligands, are 213 obtained regio- and diastereoselectively in $75-91 \%$ isolated 214 yields. In addition, the nanostructured catalyst displayed much 215 higher catalytic activity than the commercial bulk copper 216 catalysts which failed in the first step. Furthermore, simple and 217 quantitative oxidation-elimination procedures allow the trans- 218 formation of the products into 1-vinyl-4-substituted or 4- 219 monosubstituted 1,2,3-triazoles.

\section{EXPERIMENTAL SECTION}

General Methods. Anhydrous copper(II) chloride (97\%), lithium 222 powder (MEDALCHEMY S. L.), DTBB (4,4'-di-tert-butylbiphenyl), 223 activated charcoal (Norit CA1), and sodium azide were commercially 224 available. All the starting materials and other reagents were 225 commercially available of the best grade and were used without 226 further purification. THF was dried in a solvent purification system 227 using an alumina column. Melting points are uncorrected. Infrared 228 analysis was performed with a FT-IR spectrophotometer equipped 229 with an ATR component; wavenumbers are given in $\mathrm{cm}^{-1}$. NMR 230 spectra were recorded at 300 or $400 \mathrm{MHz}$ for ${ }^{1} \mathrm{H}$ NMR and 75 and 231 $101 \mathrm{MHz}$ for ${ }^{13} \mathrm{C}$ NMR; chemical shifts are given in $(\delta)$ parts per 232 million and coupling constants $(J)$ in hertz. Mass spectra (EI) were 233 obtained at $70 \mathrm{eV}$ with a GC-MS apparatus; fragment ions in $\mathrm{m} / z_{234}$ with relative intensities (\%) in parentheses. HRMS analyses were also 235 carried out in the electron impact mode (EI) at $70 \mathrm{eV}$ using a 236 quadrupole analyzer. The purity of volatile compounds and the 237 chromatographic analyses (GLC) were determined with a gas 238 chromatograph equipped with a flame ionization detector and a 30239 m capillary column ( $0.32 \mathrm{~mm}$ diameter, $0.25 \mu \mathrm{m}$ film thickness), using 240 nitrogen $(2 \mathrm{~mL} / \mathrm{min})$ as carrier gas, $T_{\text {injector }}=270{ }^{\circ} \mathrm{C}, T_{\text {column }}=60^{\circ} \mathrm{C} 241$ $(3 \mathrm{~min})$ and $60-270^{\circ} \mathrm{C}\left(15^{\circ} \mathrm{C} / \mathrm{min}\right)$; retention times $\left(t_{r}\right)$ are given in 242 min. Thin layer chromatography was carried out on TLC plastic sheets 243 with silica gel. Column chromatography was performed using silica gel 244 of 40-60 $\mu \mathrm{m}$ (hexane-EtOAc as eluent).

Typical Procedure for the Preparation of CuNPs/:. ${ }^{17 a, b} 246$ Anhydrous copper(II) chloride (135 mg, $1 \mathrm{mmol}$ ) was added to a 247 suspension of lithium (14 mg, $2 \mathrm{mmol}$ ) and 4,4'-di-tert-butylbiphenyl 248 (DTBB, $27 \mathrm{mg}, 0.1 \mathrm{mmol}$ ) in THF $(2 \mathrm{~mL}$ ) at room temperature 249 under an argon atmosphere. The reaction mixture, which was initially 250 dark blue, rapidly changed to black, indicating that the suspension of 251 copper nanoparticles was formed. This suspension was diluted with 252 THF $(18 \mathrm{~mL})$ followed by the addition of the activated carbon $\left(\begin{array}{ll}1.28 & 253\end{array}\right.$ $\mathrm{g}$ ). The resulting mixture was stirred for $1 \mathrm{~h}$ at room temperature, 254 filtered, and the solid successively washed with water $(20 \mathrm{~mL})$, THF 255 $(20 \mathrm{~mL})$, and dried under vacuum. 
257 Typical Procedure for the CuNPs/C-Catalyzed Synthesis of 258 1,2,3-Triazoles from Alkenes. $\mathrm{NaN}_{3}(39 \mathrm{mg}, 0.6 \mathrm{mmol}$ ), freshly 259 prepared dimethyl(methylthio)sulfonium tetrafluoroborate 260 (DMTSF, ${ }^{19} 118 \mathrm{mg}, 0.6 \mathrm{mmol}$ ), and cyclohexene (1a, $51 \mu \mathrm{L}, 0.5$ $261 \mathrm{mmol}$ ) were added to a suspension of CuNPs/C (10 mg, $0.5 \mathrm{~mol} \%$ $262 \mathrm{Cu})$ in $\mathrm{MeCN}(2 \mathrm{~mL})$ at room temperature under an argon 263 atmosphere. ${ }^{22}$ After the mixture was stirred for $1 \mathrm{~h}$, phenylacetylene $264(2 \mathrm{a}, 55 \mu \mathrm{L}, 0.5 \mathrm{mmol})$ was added. The reaction mixture was warmed 265 to $70{ }^{\circ} \mathrm{C}$ and monitored by TLC until total or steady conversion of the 266 starting materials. Water $(20 \mathrm{~mL})$ was added to the resulting mixture 267 followed by extraction with EtOAc $(3 \times 10 \mathrm{~mL})$. The collected organic 268 phases were dried with $\mathrm{MgSO}_{4}$, and the solvent was removed in vacuo 269 to give the corresponding triazole 3aa, which was purified by column 270 chromatography (hexane-EtOAc, 8:2).

$271 \quad 1-\left[\left(1 R^{*}, 2 R^{*}\right)\right.$-2-(Methylthio)cyclohexyl]-4-phenyl-1H-1,2,3272 triazole (3aa): pale yellow solid (110.6 mg, 81\%); mp 128.0-130.1 $273{ }^{\circ} \mathrm{C} ; t_{\mathrm{R}} 18.53 \mathrm{~min} ; R_{f} 0.61$ (hexane-EtOAc, 7:3); IR (KBr) $\nu$ 3119, 274 3082, 2935, 2923, 2850, 1480, 1460, 1435, 1211, 1178, 1076, 1048, 275 974, 762, $697 \mathrm{~cm}^{-1} ;{ }^{1} \mathrm{H}$ NMR $\left(400 \mathrm{MHz}, \mathrm{CDCl}_{3}\right) \delta 7.88-7.84(\mathrm{~m}$, $2762 \mathrm{H}), 7.81(\mathrm{~s}, 1 \mathrm{H}), 7.46-7.35(\mathrm{~m}, 2 \mathrm{H}), 7.34-7.29(\mathrm{~m}, 1 \mathrm{H}), 4.24(\mathrm{td}, J$ $277=11.2,4.2 \mathrm{~Hz}, 1 \mathrm{H}), 3.00(\mathrm{td}, J=11.2,4.2 \mathrm{~Hz}, 1 \mathrm{H}), 2.36-2.09(\mathrm{~m}$, $2783 \mathrm{H}), 1.99-1.86(\mathrm{~m}, 2 \mathrm{H}), 1.71(\mathrm{~s}, 3 \mathrm{H}), 1.56-1.42(\mathrm{~m}, 3 \mathrm{H}) ;{ }^{13} \mathrm{C} \mathrm{NMR}$ $279\left(101 \mathrm{MHz}, \mathrm{CDCl}_{3}\right) \delta 147.0,130.8,128.7,128.0,125.7,119.5,65.8$, 280 50.4, 33.9, 33.3, 25.9, 25.1, 13.8; GC-MS (EI) $m / z 273$ (26) $\left[\mathrm{M}^{+}\right]$, 281230 (27), 196 (12), 162 (14), 129 (46), 128 (68), 117 (14), 116 (22), 282102 (16), 89 (15), 81 (100), 79 (20), 61 (19); HRMS (EI) $\mathrm{m} / z$ calcd 283 for $\mathrm{C}_{15} \mathrm{H}_{19} \mathrm{~N}_{3} \mathrm{~S} 273.1300$, found 273.1293 .

2841 -[(1 $\left.R^{*}, 2 R^{*}\right)$-[2-(Methylthio)cyclohexyl]]-4-( $p$-tolyl)-1H-1,2,3285 triazole (3ab): white solid (113.4 mg, 79\%); mp $135.9-138.1{ }^{\circ} \mathrm{C}$; $t_{r}$ $28619.79 \mathrm{~min} ; R_{f} 0.54$ (hexane-EtOAc, 7:3); IR (neat) $\nu$ 3103, 2942, 287 2920, 2856, 1498, 1445, 1422, 1214, 1049, 977, $816 \mathrm{~cm}^{-1}$; ${ }^{1} \mathrm{H}$ NMR $288\left(400 \mathrm{MHz}, \mathrm{CDCl}_{3}\right) \delta 7.78(\mathrm{~s}, 1 \mathrm{H}), 7.75(\mathrm{~d}, J=8.0 \mathrm{~Hz}, 2 \mathrm{H}), 7.23(\mathrm{~d}, J$ $289=8.0 \mathrm{~Hz}, 2 \mathrm{H}), 4.23(\mathrm{td}, J=11.3,4.4 \mathrm{~Hz}, 1 \mathrm{H}), 3.00(\mathrm{td}, J=11.3,4.0$ $290 \mathrm{~Hz}, 1 \mathrm{H}), 2.38(\mathrm{~s}, 3 \mathrm{H}), 2.33-2.25(\mathrm{~m}, 1 \mathrm{H}), 2.24-2.11(\mathrm{~m}, 2 \mathrm{H}), 1.99-$ $2911.85(\mathrm{~m}, 2 \mathrm{H}), 1.70(\mathrm{~s}, 3 \mathrm{H}), 1.56-1.39(\mathrm{~m}, 3 \mathrm{H}) ;{ }^{13} \mathrm{C} \mathrm{NMR}(101 \mathrm{MHz}$, $\left.292 \mathrm{CDCl}_{3}\right) \delta 147.3,137.9,129.6,128.1,125.7,119.3,65.9,50.5,34.1$, 293 33.4, 26.0, 25.3, 21.4, 14.0; GC-MS (EI) $m / z 287(22)\left[\mathrm{M}^{+}\right], 244$ 294 (14), 131 (10), 130 (19), 129 (51), 128 (15), 115 (18), 81 (100), 79 295 (15), 77 (10), 61 (19); HRMS (EI) $\mathrm{m} / z$ calcd for $\mathrm{C}_{16} \mathrm{H}_{21} \mathrm{~N}_{3} \mathrm{~S}$ 296 287.1456, found 287.1461.

297 4-(4-Methoxyphenyl)-1-[(1R*,2R*)-[2-(methylthio)298 cyclohexyl]]-1H-1,2,3-triazole (3ac): pale yellow solid $(128.8 \mathrm{mg}$, 299 85\%); mp $132.8-135.5{ }^{\circ} \mathrm{C} ; t_{\mathrm{R}} 21.95 \mathrm{~min} ; R_{f} 0.53$ (hexane-EtOAc, 300 6:4); IR (neat) $\nu 3102,2937,2925,2857,1497,1245,1175,1030,828$, $301815 \mathrm{~cm}^{-1} ;{ }^{1} \mathrm{H}$ NMR $\left(400 \mathrm{MHz}, \mathrm{CDCl}_{3}\right) \delta 7.78(\mathrm{~d}, J=8.8 \mathrm{~Hz}, 2 \mathrm{H})$, $3027.74(\mathrm{~s}, 1 \mathrm{H}), 6.96(\mathrm{~d}, J=8.8 \mathrm{~Hz}, 2 \mathrm{H}), 4.23(\mathrm{td}, J=11.3,4.4 \mathrm{~Hz}, 1 \mathrm{H})$, $3033.84(\mathrm{~s}, 3 \mathrm{H}), 3.00(\mathrm{td}, J=11.3,4.1 \mathrm{~Hz}, 1 \mathrm{H}), 2.33-2.06(\mathrm{~m}, 3 \mathrm{H})$, $3041.98-1.84(\mathrm{~m}, 2 \mathrm{H}), 1.72(\mathrm{~s}, 3 \mathrm{H}), 1.55-1.41(\mathrm{~m}, 3 \mathrm{H}) ;{ }^{13} \mathrm{C}$ NMR $(101$ $\left.305 \mathrm{MHz} \mathrm{CDCl}_{3}\right) \delta 159.7,147.0,127.1,123.6,118.9,114.3,65.9,55.5$, 306 50.4, 34.1, 33.4, 26.0, 25.3, 13.9; GC-MS (EI) $m / z 303$ (35) $\left[\mathrm{M}^{+}\right]$, 307260 (29), 146 (16), 132 (21), 129 (59), 121 (10), 89 (13), 81 (100), 30879 (20), 61 (20); HRMS (EI) $m / z$ calcd for $\mathrm{C}_{16} \mathrm{H}_{21} \mathrm{~N}_{3} \mathrm{OS} 303.1405$, 309 found 303.1411 .

3101 - [(1 $\left.R^{*}, 2 R^{*}\right)$-[2-(Methylthio)cyclohexyl]]-4-[4311 (trifluoromethyl)phenyl]-1H-1,2,3-triazole (3ad): pale yellow 312 solid (141.6 mg, 83\%); mp 118.4-120.6 ${ }^{\circ} \mathrm{C}$; $t_{\mathrm{R}} 18.18 \mathrm{~min} ; R_{f} 0.53$ 313 (hexane-EtOAc, 7:3); IR (neat) $\nu$ 3099, 2943, 2924, 2856, 1620, 314 1329, 1158, 1123, 1105, 1065, 978, $839 \mathrm{~cm}^{-1}$; ${ }^{1} \mathrm{H}$ NMR (400 MHz, $\left.315 \mathrm{CDCl}_{3}\right) \delta 7.98(\mathrm{~d}, J=8.5 \mathrm{~Hz}, 2 \mathrm{H}), 7.91(\mathrm{~s}, 1 \mathrm{H}), 7.68(\mathrm{~d}, J=8.5 \mathrm{~Hz}$, $3162 \mathrm{H}), 4.23(\mathrm{td}, J=11.5,4.2 \mathrm{~Hz}, 1 \mathrm{H}), 3.01(\mathrm{td}, J=11.5,4.2 \mathrm{~Hz}, 1 \mathrm{H})$, $3172.36-2.11(\mathrm{~m}, 3 \mathrm{H}), 2.01-1.87(\mathrm{~m}, 2 \mathrm{H}), 1.74(\mathrm{~s}, 3 \mathrm{H}), 1.58-1.43(\mathrm{~m}$, $3183 \mathrm{H}) ;{ }^{13} \mathrm{C}$ NMR $\left(101 \mathrm{MHz}, \mathrm{CDCl}_{3}\right) \delta 145.8,134.3,130.0(\mathrm{q}, J=32.8$ $\left.319 \mathrm{~Hz}, \mathrm{CF}_{3}\right), 125.9,125.6,120.5,65.9,50.4,34.1,33.4,25.9,25.2,13.8$; $320 \mathrm{GC}-\mathrm{MS}(\mathrm{EI}) \mathrm{m} / z 341$ (4) $\left[\mathrm{M}^{+}\right], 129$ (20), 128 (100), 81 (95), 79 321 (20), 61 (22); HRMS (EI) $m / z$ calcd for $\mathrm{C}_{16} \mathrm{H}_{18} \mathrm{~F}_{3} \mathrm{~N}_{3} \mathrm{~S}$ 341.1174, 322 found 341.1180 .

323 4-Hexyl-1-[(1 $\left.R^{*}, 2 R^{*}\right)$-[2-(methylthio)cyclohexyl]]-1H-1,2,3324 triazole (3ae): pale orange solid (108.3, 77\%); mp 61.0-64.0 ${ }^{\circ} \mathrm{C} ; t_{\mathrm{R}}$ $32517.12 \mathrm{~min} ; R_{f} 0.62$ (hexane-EtOAc, 6:4); IR (neat) $\nu$ 3121, 3069, $3262924,2855,1445,1215,1152,1056,847,724 \mathrm{~cm}^{-1}$; ${ }^{1} \mathrm{H}$ NMR (400
$\left.\mathrm{MHz}, \mathrm{CDCl}_{3}\right) \delta 7.33(\mathrm{~s}, 1 \mathrm{H}), 4.16(\mathrm{td}, J=11.3,4.2 \mathrm{~Hz}, 1 \mathrm{H}), 2.94(\mathrm{td}, 327$ $J=11.3,4.2 \mathrm{~Hz}, 1 \mathrm{H}), 2.73(\mathrm{t}, J=7.7 \mathrm{~Hz}, 2 \mathrm{H}), 2.32-2.24(\mathrm{~m}, 1 \mathrm{H}), 328$ $2.19-2.05(\mathrm{~m}, 2 \mathrm{H}), 1.96-1.84(\mathrm{~m}, 2 \mathrm{H}), 1.74-1.63(\mathrm{~m}, 2 \mathrm{H}), 1.66(\mathrm{~s}, 329$ $3 \mathrm{H}), 1.52-1.25(\mathrm{~m}, 9 \mathrm{H}), 0.88(\mathrm{t}, J=6.4 \mathrm{~Hz}, 3 \mathrm{H}) ;{ }^{13} \mathrm{C}$ NMR $(101330$ $\left.\mathrm{MHz}, \mathrm{CDCl}_{3}\right) \delta 147.8,120.7,65.7,50.5,34.1,33.4,31.7,29.6,29.0,331$ 26.0, 25.8, 25.3, 22.7, 14.2, 13.9; GC-MS (EI) $m / z 281(2)\left[\mathrm{M}^{+}\right], 129332$ (21), 128 (82), 80 (14), 79 (18), 61 (22), 55 (10), 53 (10); HRMS 333 (EI) $\mathrm{m} / z$ calcd for $\mathrm{C}_{15} \mathrm{H}_{27} \mathrm{~N}_{3} \mathrm{~S} 281.1926$, found 281.1936.

$1-\left[\left(1 R^{*}, 8 R^{*}, Z\right)-8\right.$-(Methylthio)cyclooct-4-en-1-yl)]-4-phenyl- 335 1H-1,2,3-triazole (3ba): white solid (136.1 mg, 91\%); mp 111.2- 336 $113.8{ }^{\circ} \mathrm{C}$; $t_{\mathrm{R}} 21.25 \mathrm{~min} ; R_{f} 0.60$ (hexane-EtOAc, 6:4); IR (neat) $\nu 337$ 3120, 2948, 2919, 1436, 1083, 1051, 764, 712, 704, $692 \mathrm{~cm}^{-1}$; ${ }^{1} \mathrm{H} 338$ NMR $\left(300 \mathrm{MHz}, \mathrm{CDCl}_{3}\right) \delta 7.92-7.86(\mathrm{~m}, 2 \mathrm{H}), 7.83(\mathrm{~s}, 1 \mathrm{H}), 7.47-339$ $7.39(\mathrm{~m}, 2 \mathrm{H}), 7.38-7.33(\mathrm{~m}, 1 \mathrm{H}), 5.83-5.67(\mathrm{~m}, 2 \mathrm{H}), 4.86(\mathrm{td}, J=340$ 9.7, $3.4 \mathrm{~Hz}, 1 \mathrm{H}), 3.46-3.36(\mathrm{~m}, 1 \mathrm{H}), 2.79-2.22(\mathrm{~m}, 4 \mathrm{H}), 2.19-1.98341$ (m, 4H), $1.77(\mathrm{~s}, 3 \mathrm{H}) ;{ }^{13} \mathrm{C}$ NMR $\left(101 \mathrm{MHz}, \mathrm{CDCl}_{3}\right) \delta 146.8,130.7,342$ 130.5, 128.9, 128.2, 127.7, 125.9, 120.5, 65.0, 51.3, 33.8, 31.7, 25.7, 343 24.3, 14.8; GC-MS (EI) m/z 299 (9) $\left[\mathrm{M}^{+}\right], 285$ (11), 284 (54), 253344 (14), 252 (71), 156 (5), 154 (24), 148 (20), 143 (16), 117 (28), 116345 (35), 115 (12), 113 (10), 107 (38), 106 (15), 105 (17), 104 (31), 103346 (14), 102 (26), 91 (37), 90 (11), 89 (24), 81 (19), 80 (15), 79 (100), 347 78 (12), 77 (29), 74 (10), 67 (27), 65 (13), 63 (11), 61 (21), 54 (10), 348 53 (18); HRMS (EI) $m / z$ calcd for $\mathrm{C}_{17} \mathrm{H}_{21} \mathrm{~N}_{3} \mathrm{~S} 299.1456$, found 349 299.1448.

1 -[(1 $\left.R^{*}, 2 R^{*}\right)$-[4-(Methylthio)tetrahydrofuran-3-yl]]-4-phe- 351 nyl-1H-1,2,3-triazole (3ca): yellow semisolid (116.2 mg, 89\%); $t_{\mathrm{R}} 352$ $17.52 \mathrm{~min} ; R_{f} 0.49$ (hexane-EtOAc, 6:4); IR (neat) $\nu$ 3079, 2958, 353 2930, 1459, 1419, 1219, 1077, 1049, 971, 760, $699 \mathrm{~cm}^{-1} ;{ }^{1} \mathrm{H}$ NMR 354 $\left(400 \mathrm{MHz}, \mathrm{CDCl}_{3}\right) \delta 7.90(\mathrm{~s}, 1 \mathrm{H}), 7.87-7.81(\mathrm{~m}, 2 \mathrm{H}), 7.47-7.39(\mathrm{~m}, 355$ $2 \mathrm{H}), 7.34-7.31(\mathrm{~m}, 1 \mathrm{H}), 5.22(\mathrm{dd}, J=7.0,3.8 \mathrm{~Hz}, 1 \mathrm{H}), 4.51(\mathrm{dd}, J=356$ 9.8, $7.7 \mathrm{~Hz}, 1 \mathrm{H}), 4.27(\mathrm{~d}, J=4.1 \mathrm{~Hz}, 2 \mathrm{H}), 3.66(\mathrm{dd}, J=9.8,6.4 \mathrm{~Hz}, 357$ $1 \mathrm{H}), 3.52$ (ddd, $J=7.6,6.5,2.9 \mathrm{~Hz}, 1 \mathrm{H}), 2.26(\mathrm{~s}, 3 \mathrm{H}) ;{ }^{13} \mathrm{C}$ NMR $(100358$ $\left.\mathrm{MHz}, \mathrm{CDCl}_{3}\right) \delta 148.6,130.4,128.9,128.5,125.8,117.9,72.8,71.9,359$ 67.5, 51.9, 15.5; GC-MS (EI) $m / z 261$ (20) $\left[\mathrm{M}^{+}\right], 188(26), 156360$ (16), 146 (23), 145 (27), 143 (12), 130 (14), 128 (17), 118 (20), 117361 (87), 116 (94), 115 (21), 103 (20), 102 (43), 91 (20), 90 (25), 89362 (100), 77 (19), 76 (21), 75 (21), 74 (25), 71 (11), 69 (43), 68 (13), 363 64 (10), 63 (29), 62 (10), 61 (38), 54 (11), 51 (13); HRMS (EI) $\mathrm{m} / z 364$ calcd for $\mathrm{C}_{13} \mathrm{H}_{15} \mathrm{~N}_{3} \mathrm{OS} 261.0936$, found 261.0939.

1-[(1 $\left.R^{*}, 2 R^{*}\right)$-[5-(Methylthio)octan-4-yl]]-4-phenyl-1H-1,2,3- 366 triazole (3da): pale yellow solid (113.7 mg, 75\%); mp 52.5-55.3 ${ }^{\circ} \mathrm{C}$; 367 $t_{\mathrm{R}} 17.26 \mathrm{~min} ; R_{f} 0.43$ (hexane-EtOAc, 9:1); IR (neat) $\nu$ 3081, 2955, 368 2926, 2868, 1460, 1429, 1221, 1081, 976, 765, $725 \mathrm{~cm}^{-1}$; ${ }^{1} \mathrm{H}$ NMR 369 $\left(400 \mathrm{MHz}, \mathrm{CDCl}_{3}\right) \delta 7.97(\mathrm{~s}, 1 \mathrm{H}), 7.91-7.84(\mathrm{~m}, 2 \mathrm{H}), 7.46-7.39(\mathrm{~m}, 370$ $2 \mathrm{H}), 7.36-7.29(\mathrm{~m}, 1 \mathrm{H}), 4.16(\mathrm{td}, J=9.4,4.6 \mathrm{~Hz}, 1 \mathrm{H}), 2.88(\mathrm{td}, J=371$ 9.4, $4.6 \mathrm{~Hz}, 1 \mathrm{H}), 2.24-2.11(\mathrm{~m}, 1 \mathrm{H}), 2.05-1.95(\mathrm{~m}, 1 \mathrm{H}), 1.99(\mathrm{~s}, 372$ $3 \mathrm{H}), 1.64-1.54(\mathrm{~m}, 2 \mathrm{H}), 1.53-1.45(\mathrm{~m}, 1 \mathrm{H}), 1.44-1.35(\mathrm{~m}, 1 \mathrm{H}), 373$ $1.29-1.19(\mathrm{~m}, 2 \mathrm{H}), 0.93(\mathrm{t}, J=7.2 \mathrm{~Hz}, 3 \mathrm{H}), 0.91(\mathrm{t}, J=7.2 \mathrm{~Hz}, 3 \mathrm{H}) ; 374$ ${ }^{13} \mathrm{C}$ NMR $\left(101 \mathrm{MHz}, \mathrm{CDCl}_{3}\right) \delta 147.4,130.1,128.9,128.1,125.7,375$ 119.0, 65.2, 52.4, 34.5, 34.3, 20.4, 19.5, 15.6, 13.9, 13.8; GC-MS (EI) 376 $\mathrm{m} / z 303$ (28) [ $\left.\mathrm{M}^{+}\right], 260$ (14), 228 (14), 201 (64), 200 (12), 186 (12), 377 173 (15), 172 (100), 143 (21), 130 (33), 129 (16), 121 (11), 118378 (14), 117 (45), 116 (54), 115 (17), 111 (10), $110(20), 104$ (42), 103379 (73), 102 (38), 91 (62), 90 (18), 89 (42), 86 (10), 81 (10), 77 (19), 380 76 (11), 69 (75), 63 (19), 61 (96), 55 (54); HRMS (EI) $\mathrm{m} / z$ calcd for 381 $\mathrm{C}_{17} \mathrm{H}_{25} \mathrm{~N}_{3} \mathrm{~S}$ 303.1769, found 303.1759.

1-[2-(Methylthio)octyl]-4-phenyl-1H-1,2,3-triazole (3ea): pale 383 yellow solid (86.4 mg, 57\%); mp 39.8-44.4 ${ }^{\circ} \mathrm{C}$; $t_{r} 19.41 \mathrm{~min} ; R_{f} 0.66384$ (hexane-EtOAc, 7:3); IR (neat) $\nu$ 3081, 2953, 2926, 2855, 1461, 385 1435, 1224, 1084, 977, 766, 727, $694 \mathrm{~cm}^{-1} ;{ }^{1} \mathrm{H}$ NMR (300 MHz, 386 $\left.\mathrm{CDCl}_{3}\right) \delta 7.90(\mathrm{~s}, 1 \mathrm{H}), 7.88-7.81(\mathrm{~m}, 2 \mathrm{H}), 7.47-7.39(\mathrm{~m}, 2 \mathrm{H}), 387$ $7.37-7.29(\mathrm{~m}, 1 \mathrm{H}), 4.55(\mathrm{dd}, J=14.0,6.2 \mathrm{~Hz}, 1 \mathrm{H}), 4.45(\mathrm{dd}, J=14.0,388$ $7.2 \mathrm{~Hz}, 1 \mathrm{H}), 3.07-2.95(\mathrm{~m}, 1 \mathrm{H}), 1.90(\mathrm{~s}, 3 \mathrm{H}), 1.64-1.38(\mathrm{~m}, 4 \mathrm{H}), 389$ $1.33-1.20(\mathrm{~m}, 6 \mathrm{H}), 0.87(\mathrm{t}, J=6.7 \mathrm{~Hz}, 3 \mathrm{H}) ;{ }^{13} \mathrm{C}$ NMR $(75 \mathrm{MHz}, 390$ $\left.\mathrm{CDCl}_{3}\right) \delta 147.6,130.6,128.9,128.3,125.9,120.8,54.5,47.6,32.0,391$ 31.8, 29.1, 26.8, 22.7, 14.2, 13.8; GC-MS (EI) $\mathrm{m} / z 303$ (15) $\left[\mathrm{M}^{+}\right], 392$ 260 (26), 176 (20), 163 (10), 162 (13), 159 (67), 158 (11), 148 (18), 393 145 (19), 144 (24), 143 (37), 130 (26), 117 (25), $116(32), 111(20), 394$ 110 (12), 104 (31), 103 (26), 102 (29), 91 (14), 89 (26), 88 (18), 77395 
396 (18), 75 (14), 69 (93), 67 (13), 63 (14), 61 (100), 55 (79); HRMS 397 (EI) $\mathrm{m} / z$ calcd for $\mathrm{C}_{17} \mathrm{H}_{25} \mathrm{~N}_{3} \mathrm{~S} 303.1769$, found 303.1760 .

398 1-[1-(Methylthio)octan-2-yl]-4-phenyl-1H-1,2,3-triazole 399 (3ea'): yellow oil (28.8 mg, 19\%); $t_{\mathrm{R}} 19.01 \mathrm{~min} ; R_{f} 0.69$ (hexane400 EtOAc, 7:3); IR (neat) $\nu$ 2953, 2923, 2856, 1459, 1433, 1224, 762, 694 $401 \mathrm{~cm}^{-1}$; ${ }^{1} \mathrm{H}$ NMR $\left(300 \mathrm{MHz}, \mathrm{CDCl}_{3}\right) \delta 7.91-7.84(\mathrm{~m}, 2 \mathrm{H}), 7.83(\mathrm{~s}$, $4021 \mathrm{H}), 7.48-7.39(\mathrm{~m}, 2 \mathrm{H}), 7.37-7.29(\mathrm{~m}, 1 \mathrm{H}), 4.63$ (ddt, $J=8.9,7.6$, $4035.7 \mathrm{~Hz}, 1 \mathrm{H}), 3.11-2.93(\mathrm{~m}, 2 \mathrm{H}), 2.13-2.00(\mathrm{~m}, 2 \mathrm{H}), 1.95(\mathrm{~s}, 3 \mathrm{H})$, $4041.34-1.17(\mathrm{~m}, 8 \mathrm{H}), 0.85(\mathrm{t}, J=6.8 \mathrm{~Hz}, 3 \mathrm{H}) ;{ }^{13} \mathrm{C}$ NMR $(75 \mathrm{MHz}$, $\left.405 \mathrm{CDCl}_{3}\right) \delta 147.5,130.7,128.9,128.3,125.9,119.2,62.4,39.9,34.5$, 406 31.6, 28.9, 26.0, 22.6, 16.4, 14.1; GC-MS (EI) $m / z 303$ (17) $\left[\mathrm{M}^{+}\right]$, 407260 (28), 228 (12), 215 (10), 214 (63), 163 (23), 159 (36), 158 (71), 408148 (36), 144 (24), 143 (21), 130 (26), 117 (50), 116 (52), 104 (43), 409103 (31), 102 (30), 91 (35), 90 (16), 89 (32), 77 (12), 75 (10), 69 410 (86), 67 (13), 63 (15), 61 (100), 55 (60); HRMS (EI) $\mathrm{m} / z$ calcd for $411 \mathrm{C}_{17} \mathrm{H}_{25} \mathrm{~N}_{3} \mathrm{~S} 303.1769$, found 303.1779 .

$412 \quad 1-\left[\left(1 R^{*}, 2 R^{*}\right)\right.$-2-Methyl-2-(methylthio)cyclohexyl]-4-phenyl$4131 \mathrm{H}-1,2,3$-triazole (3fa): pale yellow solid (53.1 mg, 37\%); mp 78.9$41481.9^{\circ} \mathrm{C}$; $t_{\mathrm{R}} 19.89 \mathrm{~min} ; R_{f} 0.53$ (hexane-EtOAc, 7:3); IR (KBr) $\nu$ 3117, 415 2934, 2858, 1481, 1458, 1434, 1387, 1228, 1077, 979, 764, $697 \mathrm{~cm}^{-1}$; $416{ }^{1} \mathrm{H}$ NMR (400 MHz, $\left.\mathrm{CDCl}_{3}\right) \delta 7.87-7.84(\mathrm{~m}, 2 \mathrm{H}), 7.83(\mathrm{~s}, 1 \mathrm{H})$, $4177.45-7.40(\mathrm{~m}, 2 \mathrm{H}), 7.35-7.30(\mathrm{~m}, 1 \mathrm{H}), 4.54(\mathrm{dd}, J=12.0,3.9 \mathrm{~Hz}$, $4181 \mathrm{H}), 2.39-2.29(\mathrm{~m}, 1 \mathrm{H}), 2.09-2.03(\mathrm{~m}, 1 \mathrm{H}), 2.01-1.95(\mathrm{~m}, 2 \mathrm{H})$, $4191.86(\mathrm{~s}, 3 \mathrm{H}), 1.81-1.73(\mathrm{~m}, 2 \mathrm{H}), 1.72-1.66(\mathrm{~m}, 2 \mathrm{H}), 1.35(\mathrm{~s}, 3 \mathrm{H})$; ${ }_{420}{ }^{13} \mathrm{C}$ NMR $\left(101 \mathrm{MHz}, \mathrm{CDCl}_{3}\right) \delta 146.7,130.9,128.9,128.1,125.8$, 421 120.6, 67.0, 48.1, 38.7, 29.0, 25.4, 21.9, 19.2, 10.8; GC-MS (EI) $m / z$ $422287(56)\left[\mathrm{M}^{+}\right], 288(10)\left[\mathrm{M}^{+}+1\right], 244(18), 212(21), 146(22), 145$ 423 (14), 143 (49), 117 (17), 116 (25), 102 (18), 99 (14), 96 (13), 95 424 (100), 93 (10), 91 (13), 77 (11), 75 (11), 67 (23), 55 (16); HRMS 425 (EI) $m / z$ calcd for $\mathrm{C}_{16} \mathrm{H}_{21} \mathrm{~N}_{3} \mathrm{~S} 287.1456$, found 287.1462 .

4261 -[(1 $\left.R^{*}, 2 R^{*}\right)$-1-Methyl-2-(methylthio)cyclohexyl]-4-phenyl$4271 \mathrm{H}$-1,2,3-triazole (3fa'): yellow oil $(60.3 \mathrm{mg}, 42 \%) ; t_{\mathrm{R}} 18.88 \mathrm{~min} ; R_{f}$ 4280.59 (hexane-EtOAc, 7:3); IR (neat) ข 3130, 2928, 2862, 1458, 1448, $4291234,1025,765,699 \mathrm{~cm}^{-1}$; ${ }^{1} \mathrm{H}$ NMR $\left(400 \mathrm{MHz}, \mathrm{CDCl}_{3}\right) \delta 7.93(\mathrm{~s}$, $4301 \mathrm{H}), 7.89-7.84(\mathrm{~m}, 2 \mathrm{H}), 7.45-7.39(\mathrm{~m}, 2 \mathrm{H}), 7.35-7.29(\mathrm{~m}, 2 \mathrm{H})$, $4313.42(\mathrm{dd}, J=11.9,4.0 \mathrm{~Hz}, 1 \mathrm{H}), 2.64-2.54(\mathrm{~m}, 1 \mathrm{H}), 2.16-2.12(\mathrm{~m}$, $4322 \mathrm{H}), 2.01-1.94(\mathrm{~m}, 1 \mathrm{H}), 1.87-1.77(\mathrm{~m}, 2 \mathrm{H}), 1.75(\mathrm{~s}, 3 \mathrm{H}), 1.69(\mathrm{~s}$, $4331 \mathrm{H}), 1.66-1.53(\mathrm{~m}, 3 \mathrm{H}) ;{ }^{13} \mathrm{C} \mathrm{NMR}\left(101 \mathrm{MHz}, \mathrm{CDCl}_{3}\right) \delta 146.6$, 434 131.0, 128.9, 128.0, 125.7, 118.4, 66.3, 55.9, 39.4, 31.3, 25.9, 22.4, 19.1, 435 15.9; GC-MS (EI) $m / z 287$ (9) $\left[\mathrm{M}^{+}\right], 244$ (11), 212 (12), 144 (10), 436143 (35), 142 (98), 117 (16), 116 (14), 102 (11), 96 (10), 95 (100), 43767 (20), 61 (10), 55 (11); HRMS (EI) $m / z$ calcd for $\mathrm{C}_{16} \mathrm{H}_{21} \mathrm{~N}_{3} \mathrm{~S}$ 438287.1456 , found 287.1460 .

439 1-[2-(Methylthio)-1-phenylethyl]-4-phenyl-1 $\mathrm{H}$-1,2,3-triazole 440 (3ga): white solid (131.3 mg, 89\%); mp 115.5-118.2 ${ }^{\circ} \mathrm{C}$; $t_{\mathrm{R}} 19.24$ $441 \mathrm{~min} ; R_{f} 0.62$ (hexane-EtOAc, 6:4); IR (neat) $\nu$ 3083, 2921, 2909, 442 1456, 1436, 1219, 1077, 763, 708, 700, $689 \mathrm{~cm}^{-1}$; ${ }^{1} \mathrm{H}$ NMR (300 $\left.443 \mathrm{MHz}, \mathrm{CDCl}_{3}\right) \delta 7.87-7.79(\mathrm{~m}, 2 \mathrm{H}), 7.75(\mathrm{~s}, 1 \mathrm{H}), 7.49-7.29(\mathrm{~m}, 8 \mathrm{H})$, $4445.74(\mathrm{dd}, J=8.2,6.7 \mathrm{~Hz}, 1 \mathrm{H}), 3.68(\mathrm{dd}, J=14.0,8.2 \mathrm{~Hz}, 1 \mathrm{H}), 3.37$ $445(\mathrm{dd}, J=14.0,6.7 \mathrm{~Hz}, 1 \mathrm{H}), 2.03(\mathrm{~s}, 3 \mathrm{H}) ;{ }^{13} \mathrm{C}$ NMR $\left(75 \mathrm{MHz}^{\mathrm{CDCl}}{ }_{3}\right)$ $446 \delta 147.8,137.8,130.6,129.3,129.2,128.9,128.3,127.3,125.8,119.6$, 447 66.6, 39.3, 16.5; GC-MS (EI) $m / z 295$ (7) $\left[\mathrm{M}^{+}\right], 234$ (26), 207 (14), 448206 (74), 204 (14), 179 (10), 178 (31), 163 (15), 152 (13), 151 (60), 449150 (100), 148 (15), 145 (28), 137 (12), 136 (30), 135 (47), 134 450 (16), 128 (12), 118 (10), 117 (20), $116(51), 105$ (11), 104 (59), 103 451 (45), 102 (27), 91 (45), 90 (13), 89 (37), 78 (15), 77 (42), 76 (13), 45263 (21), 61 (14), 51 (18); HRMS (EI) $m / z$ calcd for $\mathrm{C}_{17} \mathrm{H}_{17} \mathrm{~N}_{3} \mathrm{~S}$ 453295.1143 , found 295.1137 .

454 4-Hexyl-1-[2-(methylthio)-1-phenylethyl]-1 H-1,2,3-triazole

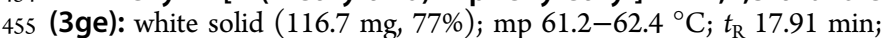
$456 R_{f} 0.69$ (hexane-EtOAc, 6:4); IR (neat) $\nu$ 3113, 3064, 2954, 2919, $4572854,1457,1429,1058,851,747,704 \mathrm{~cm}^{-1}$; ${ }^{1} \mathrm{H}$ NMR $(300 \mathrm{MHz}$, $\left.458 \mathrm{CDCl}_{3}\right) \delta 7.39-7.33(\mathrm{~m}, 5 \mathrm{H}), 7.28(\mathrm{~s}, 1 \mathrm{H}), 5.66(\mathrm{dd}, J=8.2,6.7 \mathrm{~Hz}$, $4591 \mathrm{H}), 3.60(\mathrm{dd}, J=14.0,8.2 \mathrm{~Hz}, 1 \mathrm{H}), 3.31(\mathrm{dd}, J=14.0,6.7 \mathrm{~Hz}, 1 \mathrm{H})$, $4602.70(\mathrm{t}, J=7.7 \mathrm{~Hz}, 2 \mathrm{H}), 1.99(\mathrm{~s}, 3 \mathrm{H}), 1.70-1.56(\mathrm{~m}, 2 \mathrm{H}), 1.38-1.24$ $461(\mathrm{~m}, 6 \mathrm{H}), 0.86(\mathrm{t}, J=6.8 \mathrm{~Hz}, 3 \mathrm{H}) ;{ }^{13} \mathrm{C}$ NMR $\left(75 \mathrm{MHz}, \mathrm{CDCl}_{3}\right) \delta$ $462148.4,138.1,129.2,129.0,127.3,120.7,65.3,39.4,31.6,29.4,29.0$, $46325.8,22.7,16.3,14.2$; GC-MS (EI) $m / z 303$ (1) $\left[\mathrm{M}^{+}\right], 214(24), 151$ 464 (49), 150 (100), 144 (12), 136 (21), 135 (35), 134 (13), 104 (40),
103 (25), 96 (10), 91 (43), 83 (13), 77 (15); HRMS (EI) $m / z$ calcd 465 for $\mathrm{C}_{17} \mathrm{H}_{25} \mathrm{~N}_{3} \mathrm{~S} 303.1769$, found 303.1767 .

1-[(Methylsulfinyl)(phenyl)methyl]-4-phenyl-1 H-1,2,3-tria- 467 zole (4ga). In a typical procedure, ${ }^{23}$ a round-bottom flask was charged 468 with sulfide 3 ga $(50.7 \mathrm{mg}, 0.17 \mathrm{mmol}), \mathrm{MeOH}(1 \mathrm{~mL})$, and the 469 catalyst $\left[0.1 \mathrm{~mL}\right.$ of a solution prepared by mixing $96 \% \mathrm{H}_{2} \mathrm{SO}_{4}(1.38 \mathrm{~g}) 470$ and 2-propanol $(38 \mathrm{~mL})] . \mathrm{H}_{2} \mathrm{O}_{2}(0.05 \mathrm{~mL}, 0.50 \mathrm{mmol})$ was added at 471 once to the stirred mixture, and the progress of the oxidation was 472 followed by TLC $(1-2 \mathrm{~h})$. Water $(10 \mathrm{~mL})$ was added to the mixture 473 after completion of the reaction. The aqueous phase was saturated 474 with $\mathrm{NaCl}$ and extracted with EtOAc $(3 \times 10 \mathrm{~mL})$. The organic phase 475 was dried with $\mathrm{MgSO}_{4}$ and evaporated to give the pure sulfoxide 4ga 476 $(53.0 \mathrm{mg}, 99 \%)$ as a ca. 1:1 diastereomeric mixture: white solid; $\mathrm{mp} 477$ 133.9-135.4 ${ }^{\circ} \mathrm{C}$; $R_{f} 0.34$ (EtOAc); IR (KBr) $\nu$ 3080, 2926, 1457, 478 1432, 1032, 1023, 975, 763, 714, $690 \mathrm{~cm}^{-1} ;{ }^{1} \mathrm{H}$ NMR $(300 \mathrm{MHz}, 479$ $\left.\mathrm{CDCl}_{3}\right) \delta 7.91(\mathrm{~s}, 1 \mathrm{H}), 7.85(\mathrm{~s}, 1 \mathrm{H}), 7.84-7.87(\mathrm{~m}, 16 \mathrm{H}), 6.20-6.11480$ $(\mathrm{m}, 2 \mathrm{H}), 4.30(\mathrm{t}, J=12.6 \mathrm{~Hz}, 2 \mathrm{H}), 4.03(\mathrm{dd}, J=13.2,5.8 \mathrm{~Hz}, 2 \mathrm{H}), 481$ 3.79 (dd, J = 13.2, $8.7 \mathrm{~Hz}, 2 \mathrm{H}), 3.34$ (dd, $J=13.2,2.9 \mathrm{~Hz}, 2 \mathrm{H}), 2.70482$ (s, 3H), $2.55(\mathrm{~s}, 3 \mathrm{H}) ;{ }^{13} \mathrm{C} \mathrm{NMR}\left(75 \mathrm{MHz}, \mathrm{CDCl}_{3}\right) \delta 148.2,137.6,483$ 136.9, 130.0, 129.9, 129.8, 129.7, 129.6, 129.5, 129.4, 129.0, 128.6, 484 128.6, 127.3, 126.9, 126.8, 125.9, 121.2, 120.5, 60.2, 59.5, 58.8, 58.0, 485 39.2, 38.5; GC-MS (EI) $m / z 311(2)\left[\mathrm{M}^{+}\right], 249$ (10), 248 (57), 219486 (10), 167 (10), 151 (22), 117 (13), 116 (100), 105 (13), 104 (77), 487 103 (23), 91 (13), 89 (21), 77 (14); HRMS (EI) $\mathrm{m} / z$ calcd for 488 $\mathrm{C}_{17} \mathrm{H}_{17} \mathrm{~N}_{3} \mathrm{OS} 311.1092$, found 311.1095 .

4-Phenyl-1-(1-phenylvinyl)-1H-1,2,3-triazole (5ga). In a typi- 490 cal procedure, the sulfoxide $4 \mathrm{ga}(29.5 \mathrm{mg}, 0.1 \mathrm{mmol})$ was heated in 491 toluene at $120{ }^{\circ} \mathrm{C}$ for $10 \mathrm{~h}$ in a pressure tube with a Teflon cap. 492 Evaporation of the solvent gave the pure triazole $5 \mathrm{ga}(22.0 \mathrm{mg}, 90 \%) 493$ as a yellow oil. The physical and spectroscopic data of 5ga were 494 compared with those reported in the literature: ${ }^{21 \mathrm{c}}{ }^{1} \mathrm{H}$ NMR (300 495 $\left.\mathrm{MHz}, \mathrm{CDCl}_{3}\right) \delta 7.88-7.83(\mathrm{~m}, 2 \mathrm{H}), 7.80(\mathrm{~s}, 1 \mathrm{H}), 7.48-7.34(\mathrm{~m}, 8 \mathrm{H}), 496$ $5.88(\mathrm{~d}, J=1.0 \mathrm{~Hz}, 1 \mathrm{H}), 5.57(\mathrm{~d}, J=1.0 \mathrm{~Hz}, 1 \mathrm{H}) ;{ }^{13} \mathrm{C} \mathrm{NMR}(75 \mathrm{MHz}, 497$ $\left.\mathrm{CDCl}_{3}\right) \delta 147.7,143.1,134.8,130.3,130.1,129.0,128.5,127.5,125.9,498$ $119.9,109.6$.

1-[(Methylsulfonyl)(phenyl)methyl]-4-phenyl-1H-1,2,3-tria- 500 zole (6ga). In a typical procedure, ${ }^{24}$ a solution of $m$-chloroperbenzoic 501 acid $(86.3 \mathrm{mg}, 0.50 \mathrm{mmol})$ in $\mathrm{CH}_{2} \mathrm{Cl}_{2}(2 \mathrm{~mL})$ was added to a solution 502 of triazole $3 \mathrm{ga}(29.5 \mathrm{mg}, 0.1 \mathrm{mmol})$ in $\mathrm{CH}_{2} \mathrm{Cl}_{2}(2 \mathrm{~mL})$ at $0{ }^{\circ} \mathrm{C}$; the 503 reaction was stirred at $0{ }^{\circ} \mathrm{C}$ for $2 \mathrm{~h}$. Then, it was quenched with 504 saturated aqueous sodium bicarbonate $(10 \mathrm{~mL})$ and diluted with 505 $\mathrm{CH}_{2} \mathrm{Cl}_{2}(10 \mathrm{~mL})$. The organic layer was removed and the aqueous 506 layer was extracted with $\mathrm{CH}_{2} \mathrm{Cl}_{2}(10 \mathrm{~mL})$. The combined organic 507 layers were dried with $\mathrm{MgSO}_{4}$, the solvent was evaporated, and the 508 crude mixture was purified by column chromatography (silica gel, 509 hexane-EtOAc, 3:7)] to give the sulfone $6 \mathrm{ga}(28.4 \mathrm{mg}, 87 \%)$ as a white 510 solid; $\mathrm{mp} 164.7-167.7{ }^{\circ} \mathrm{C}$; $t_{\mathrm{R}} 21.85 \mathrm{~min} ; R_{\mathrm{f}} 0.52$ (hexane-EtOAc, 511 1:1); IR (KBr) レ 3093, 2923, 1335, 1302, 1149, 1129, 1051, 1088, 512 $1051,976,747,696,669 \mathrm{~cm}^{-1}$; ${ }^{1} \mathrm{H}$ NMR $\left(400 \mathrm{MHz}, \mathrm{CDCl}_{3}\right) \delta 7.88513$ (s, 1H), 7.84-7.71 (m, 2H), 7.46-7.32 (m, 8H), 6.14 (dd, J = 9.7, 4.0514 $\mathrm{Hz}, 1 \mathrm{H}), 4.78(\mathrm{dd}, J=15.2,9.7 \mathrm{~Hz}, 1 \mathrm{H}), 3.74(\mathrm{dd}, J=15.2,4.0 \mathrm{~Hz}, 515$ $1 \mathrm{H}), 2.56(\mathrm{~s}, 3 \mathrm{H}) ;{ }^{13} \mathrm{C}$ NMR $\left(101 \mathrm{MHz}, \mathrm{CDCl}_{3}\right) \delta 148.6,136.7,516$ 129.8, 129.8, 129.7, 129.1, 128.8, 126.9, 125.9, 120.8, 60.8, 59.5, 42.4; 517 GC-MS (EI) $m / z 327$ (5) [M+], 207 (23), 206 (12), 183 (13), 117518 (15), 116 (100), 105 (11), 104 (54), 103 (16), 102 (10), 91 (16), 89519 (17), 77 (11); HRMS (EI) $\mathrm{m} / z$ calcd for $\mathrm{C}_{17} \mathrm{H}_{17} \mathrm{~N}_{3} \mathrm{O}_{2} \mathrm{~S} 327.1041,520$ found 327.1042 .

4-Phenyl-1H-1,2,3-triazole (7a) and (E)-[2-(methylsulfonyl)- 522 vinyl]benzene (8a). In a typical procedure, ${ }^{25}$ a solution of compound 523 6 ga $(50.0 \mathrm{mg}, 0.15 \mathrm{mmol})$ in dry $\mathrm{MeOH}(1 \mathrm{~mL})$ and THF $(0.5 \mathrm{~mL}) 524$ was added to a stirred suspension of $\mathrm{Na} / \mathrm{Hg}$ [freshly prepared from $\mathrm{Na} 525$ (70.0 mg, $3.0 \mathrm{mmol})$ and $\mathrm{Hg}(1.163 \mathrm{~g}, 5.8 \mathrm{mmol})]$ and $\mathrm{Na}_{2} \mathrm{HPO}_{4} 526$ $(428 \mathrm{mg}, 3.0 \mathrm{mmol})$ in $\mathrm{MeOH}(2 \mathrm{~mL})$ under argon. The reaction 527 progress was monitored by TLC and GLC. The mixture was then 528 filtered, and the filter cake was washed with $\mathrm{Et}_{2} \mathrm{O}$. The combined 529 filtrate was evaporated under vacuum and purified by preparative TLC 530 (hexane-EtOAc, $1: 1)$ to give triazole $7 \mathbf{a}(21.7 \mathrm{mg}$, 99\%) and vinyl 531 sulfone $8 \mathrm{a}(27.3 \mathrm{mg}, 99 \%)$ as colorless solids in quantitative yields. 532 The physical and spectroscopic data of $7 a^{26}$ and $8 a^{27}$ were in 533 agreement with those reported in the literature. 


\section{$535 \square$ ASSOCIATED CONTENT}

\section{S Supporting Information}

$537{ }^{1} \mathrm{H}$ and ${ }^{13} \mathrm{C}$ NMR spectra and some GLC-MS analyses. This 538 material is available free of charge via the Internet at http:// 539 pubs.acs.org.

\section{$540 \square$ AUTHOR INFORMATION}

\section{Corresponding Author}

542 *E-mail: falonso@ua.es.

\section{Notes}

544 The authors declare no competing financial interest.

\section{$545 \square$ ACKNOWLEDGMENTS}

546 This work was generously supported by the Spanish Ministerio 547 de Economía y Competitividad (MINECO; CTQ2007-65218, 548 CTQ2011-24151 and Consolider Ingenio 2010-CSD2007549 00006), the Generalitat Valenciana (GV; PROMETEO/ 550 2009/039), and Fondo Europeo de Desarrollo Regional 551 (FEDER). Y.M. acknowledges the Instituto de Sintesis 552 Orgánica (ISO) of the Universidad de Alicante for a grant.

\section{$553 \square$ REFERENCES}

554 (1) For a review, see: Kolb, H. C.; Finn, M. G.; Sharpless, K. B. 555 Angew. Chem., Int. Ed. 2001, 40, 2004.

556 (2) (a) Wang, Q.; Hawker, C. Chem. Asian J. 2011, 6, 2568. (b) For 557 a recent special issue, see: Chem. Asian J. 2011, 6 (10).

558 (3) (a) Tornøe, C. W.; Christensen, C.; Meldal, M. J. Org. Chem. 559 2002, 67, 3057. (b) Rostovtsev, V. V.; Green, L. G.; Fokin, V. V.; 560 Sharpless, K. B. Angew. Chem., Int. Ed. 2002, 41, 2596.

561 (4) For selected reviews, see: (a) Meldal, M.; Tornøe, C. W. Chem. 562 Rev. 2008, 108, 2952. (b) Fokin, V. V. In Organic Chemistry. 563 Breakthroughs and Perpectives, 1st ed.; Ding, K., Dai, L.-X., Eds.; Wiley$564 \mathrm{VCH}$ : Weinheim, 2012; Chapter 7.

565 (5) For some recent examples, see: (a) Kumar, D.; Patel, G.; Buchi 566 Reddy, V. Synlett 2009, 399. (b) Bénéteau, V.; Olmos, A.; Boningari, 567 T.; Sommer, J.; Pale, P. Tetrahedron Lett. 2010, 51, 3673. (c) Yan, J.; 568 Wang, L. Synthesis 2010, 447. (d) Shamim, T.; Paul, S. Catal. Lett. $5692010,136,260$.

570 (6) (a) Zhang, F.; Moses, J. E. Org. Lett. 2009, 11, 1587 and 571 references cited therein. (b) Smith, N. M.; Greaves, M. J.; Jewell, R.; 572 Perry, M. W. D.; Stocks, M. J.; Stonehouse, J. P. Synlett 2009, 1391. 573 (c) Suárez, J. R.; Trastoy, B.; Pérez Ojeda, E.; Marín-Barrios, R.; 574 Chiara, J. L. Adv. Synth. Catal. 2010, 352, 2515.

575 (7) (a) Surendra Reddy, P.; Sreedhar, B. Synthesis 2009, 4203. 576 (b) Kumar, D.; Buchi Reddy, V.; Varma, R. S. Tetrahedron Lett. 2009, 577 50, 2065.

578 (8) Kumar, D.; Buchi Reddy, V. Synthesis 2010, 1687.

579 (9) See, for instance: (a) Kumaraswamy, G.; Ankamma, K.; Pitchaiah, 580 A. J. Org. Chem. 2007, 72, 9822. (b) Rajender Reddy, K.; Uma 581 Maheswari, C.; Rajgopal, K.; Lakshmi Kantam, M. Synth. Commun. 582 2008, 38, 2158. (c) Sharghi, H.; Beyzavi, M. H.; Safavi, A.; 583 Doroodmand, M. M.; Khalifeh, R. Adv. Synth. Catal. 2009, 351, 584 2391. (d) Boningari, T.; Olmos, A.; Reddy, B. M.; Sommer, J.; Pale, P. 585 Eur. J. Org. Chem. 2010, 6338.

586 (10) Zhang, J.; Wu, J.; Shen, L.; Cao, S. Adv. Synth. Catal. 2011, 353, 587580 .

588 (11) (a) Tao, C.-Z.; Cui, X.; Li, J.; Liu, A.-X.; Liu, L.; Guo, Q.-X. 589 Tetrahedron Lett. 2007, 48, 3525. (b) Mohammed, S.; Padala, A. K.; 590 Dar, B. A.; Singh, B.; Sreedhar, B.; Vishwakarma, R. A.; Bharate, S. B. 591 Tetrahedron 2012, 68, 8156.

592 (12) Attanasi, O. A.; Favi, G.; Filippone, P.; Mantellini, F.; Moscatelli, 593 G.; Perrulli, F. R. Org. Lett. 2010, 12, 468.

594 (13) For a review, see: Jin, T.; Yan, M.; Yamamoto, Y. ChemCatChem 595 2012, 4, 1217.

596 (14) For a recent review, see: Alonso, F.; Riente, P.; Yus, M. Acc. 597 Chem. Res. 2011, 44, 379.
(15) (a) Alonso, F.; Vitale, C.; Radivoy, G.; Yus, M. Synthesis 2003, 598 443. (b) Alonso, F.; Moglie, Y.; Radivoy, G.; Vitale, C.; Yus, M. Appl. 599 Catal. A: Gen. 2004, 271, 171. (c) Radivoy, G.; Alonso, F.; Moglie, Y.; 600 Vitale, C.; Yus, M. Tetrahedron 2005, 61, 3859.

(16) (a) Alonso, F.; Moglie, Y.; Radivoy, G.; Yus, M. Tetrahedron 602 Lett. 2009, 50, 2358. (b) Alonso, F.; Moglie, Y.; Radivoy, G.; Yus, M. 603 Eur. J. Org. Chem. 2010, 1875.

(17) (a) Alonso, F.; Moglie, Y.; Radivoy, G.; Yus, M. Adv. Synth. 605 Catal. 2010, 352, 3208. (b) Alonso, F.; Moglie, Y.; Radivoy, G.; Yus, 606 M. Org. Biomol. Chem. 2011, 9, 6385. (c) Alonso, F.; Moglie, Y.; 607 Radivoy, G.; Yus, M. J. Org. Chem. 2011, 76, 8394. (d) Alonso, F.; 608 Moglie, Y.; Radivoy, G.; Yus, M. Heterocycles 2012, 84, 1033.

(18) Trost, B. M.; Shibata, T. J. Am. Chem. Soc. 1982, 104, 3225.610

(19) (a) Helmkamp, G. K.; Olsen, B. A.; Pettitt, D. J. J. Org. Chem. 611 1965, 30, 676. (b) Helmkamp, G. K.; Cassey, H. N.; Olsen, B. A.; 612 Pettitt, D. J. J. Org. Chem. 1965, 30, 933.

(20) See, for instance: (a) Zhu, Y. W.; Yi, W.-B.; Cai, C. Catal. 614 Commun. 2011, 15, 118. (b) Michaels, H. A.; Zhu, L. Chem. Asian J. 615 2011, 6, 2825.

(21) (a) L’Abbé, G.; Hassner, A. J. Heterocycl. Chem. 1970, 7, 361. 617 (b) Duan, H.; Yan, W.; Sengupta, S.; Shi, X. Bioorg. Med. Chem. Lett. 618 2009, 19, 3899. (c) Kupracz, L.; Hartwig, J.; Wegner, J.; Ceylan, S.; 619 Kirschning, A. Beilstein J. Org. Chem. 2011, 7, 1441.

(22) The argon atmosphere was used as a precautionary measure 621 because of gas evolution during the reaction (the flash point of $\mathrm{Me}_{2} \mathrm{~S}$ is 622 $-36{ }^{\circ} \mathrm{C}$ ). The reaction also proceeds under air. 623

(23) Drabowicz, J.; Lyzwa, P.; Popielarczk, M.; Mikolajczyk, M. 624 Synthesis 1990, 937.

(24) Evans, D. A.; Faul, M. M.; Colombo, L.; Bisaha, J. J.; Clardy, J.; 626 Cherry, D. J. Am. Chem. Soc. 1992, 114, 5977.

(25) Enders, D.; Jandeleit, B.; Prokopenko, O. F. Tetrahedron 1995, 628 $51,6273$.

(26) Kim, J. D.; Palani, T.; Kumar, M. R.; Lee, S.; Choi, H. C. J. 630 Mater. Chem. 2012, 22, 20665.

(27) Yuan, G.; Zheng, J.; Gao, X.; Li, X.; Huang, L.; Chen, H.; Jiang, 632 H. Chem. Commun. 2012, 48, 7513. 